\title{
TanSat ACGS on-orbit spectral calibration by use of individual solar lines and entire atmospheric spectra
}

\author{
Yanmeng Bi ${ }^{1}$, Qian Wang ${ }^{1}$, Zhongdong Yang ${ }^{1}$, Chengbao Liu ${ }^{1}$, Chao Lin ${ }^{2}$, Longfei Tian ${ }^{3}$, \\ Naiqiang Zhang ${ }^{4}$, Yanping $\mathrm{Luo}^{4}$, and Yacheng Wang ${ }^{5}$ \\ ${ }^{1}$ National Satellite Meteorological Center, CMA, Beijing, China \\ ${ }^{2}$ Changchun Institute of Optics, Fine Mechanics and Physics, CAS, Changchun, China \\ ${ }^{3}$ Innovation Academy for Microsatellites of CAS, Shanghai, China \\ ${ }^{4}$ HuaYun ShineTek, Beijing, China \\ ${ }^{5}$ Space Star Technology co., LTD, China
}

Correspondence: Qian Wang(qwang@cma.cn)

\begin{abstract}
.
The spectra measured by the Atmospheric Carbon dioxide Grating Spectrometer (ACGS) carried by the China's global carbon dioxide observation satellite (TanSat) in the band of $0.76 \mu \mathrm{m}, 1.61 \mu \mathrm{m}$ and $2.06 \mu \mathrm{m}$ can be used for the retrieval of carbon dioxide $\left(\mathrm{CO}_{2}\right)$ concentrations by fitting the observations and simulations using the optimal estimation algorithm. Accurately detecting the change of the center wavelength is highly important because of its very high spectral resolution and accuracy requirement for product retrieval. The variations of center wavelength for all three bands of ACGS have been calculated on the locations of the individual solar absorption lines by comparing the solar-viewing measurements and the high resolution solar reference spectrum. The variations with magnitudes less than $10 \%$ of the spectral resolution for each band have been detected. The changes are probably caused by vibration and the instrument status difference between the ground and space, especially temperature variation on orbit. In addition to solar lines, the entire atmospheric spectra simulated by radiative transfer model can be used as the reference spectrum to determine the wavelength change by fitting the measured and simulated spectra. The change of wavelength determined by atmospheric spectra is closely consistent with that by solar lines. Both schemes described here can be used not only for monitoring spectral stability but also to gain spectral knowledge prior to the level-2 product processing. These minor temporal changes of wavelength on orbit should be corrected in the product retrieval.
\end{abstract}

\section{Introduction}

Measuring the distribution of carbon dioxide $\left(\mathrm{CO}_{2}\right)$ is very important not only for inferring the source and sink of carbon but also for improving understanding of climate change at a more higher level (Miller et al., 2007; Chatterjee et al., 2017; Schwandner et al., 2017). The China's global carbon dioxide observation satellite (TanSat) was successfully launched on 21 December 2016 (UTC) into a sun-synchronous, $700 \mathrm{~km}$ altitude polar orbit with a 13:30 ascending local time. The main objective is to measure the distribution of $\mathrm{CO}_{2}$ with precisions of better than $1 \%$ (1-4 ppm) on regional to continental scales. The Atmospheric Carbon dioxide Grating Spectrometer (ACGS) carried by the TanSat is a three-band imaging grating spectrometer 
collecting reflected sunlight in spectra ranges around $0.76 \mu \mathrm{m}\left(\mathrm{O}_{2}\right.$ A-band, $\left.\mathrm{O} 2 \mathrm{~A}\right), 1.61 \mu \mathrm{m}$ (weak $\mathrm{CO}_{2}$ band, WCO2) and $2.06 \mu \mathrm{m}$ (strong $\mathrm{CO}_{2}$ band, $\mathrm{SCO} 2$ ). The ACGS instrument collects nine soundings at a sampling rate of $3.3 \mathrm{~Hz}$, yielding field of view (FOV) with a resolution of approximately $2 \mathrm{~km} \times 3 \mathrm{~km}$ along its ground track and cross track and a swath of $20 \mathrm{~km}$ at nadir (Yang et al., 2019).

The spectra measured by the ACGS instrument contain information on the concentration of $\mathrm{CO}_{2}$ in the atmosphere, as well as of aerosols and clouds. Because clouds shield part of the $\mathrm{CO}_{2}$ column, they must be detected for the scene of the ground observations. Cloud fraction and surface pressure are estimated from $\mathrm{O}_{2}$ absorption features in the reflected spectra. The column-average dry-air mole fraction of $\mathrm{CO}_{2}$, called $\mathrm{X}_{\mathrm{CO}_{2}}$, mainly retrieved from the WCO2 and SCO2 band by fitting the observed spectra and the simulated spectra generated by radiative transfer model (O'Dell et al., 2012; Crisp et al., 2012). In the fitting procedure, there could be large residuals resulting in failure of $\mathrm{X}_{\mathrm{CO}_{2}}$ retrieval if the observed spectra is displaced due to instrumental effects and Doppler effects. Doppler effects can be calculated from the satellite velocity. Therefore, estimating the displacement induced by instrumental effects is very essential for $\mathrm{X}_{\mathrm{CO}_{2}}$ retrieval.

On-orbit spectral calibration involves two parts that are the instrument line shape (ILS) calibration and wavelength calibration. The ILS represents the response of a single detector pixel to any wavelength of light incident upon that pixel. Sun et al. (2017) presented an excellent analysis of the on-orbit ILS variation of the OCO-2 instrument by applying different analytical functional forms to fit the ILS function. But for TanSat, those analytical function can not fully represent the ILS very well, especially at the top and the wings of the ILS where there are some irregular fine structures (see Figure 1). Therefore, we assume the ILS remains constant on orbit and only study the wavelength offset or shift with respect to pre-launch spectral calibration. To a large extent, this assumption is correct for TanSat ACGS instrument because a common diffuser is used for solar observation, which is different from transmissive diffuser used by OCO-2 (Crisp et al., 2017). In addition, there are no on-orbit decon events that can broaden the wings of the ILS (Sun et al., 2017). Therefore, this study only focuses on the wavelength calibration with an assumption of unchanged ILS.

Previous spectral calibration for grating spectrometers typically use the fitting algorithm to fit the measured solar irradiance with a solar reference spectrum (Chance, 1998; Liu et al., 2005; Liu et al., 2010; Munro et al., 2016; Sun et al., 2017). Here, we have used two types of on-orbit methods to evaluate the ACGS's wavelength calibration connecting each focal plane array (FPA) pixel to a specific wavelength. The first method is to use the solar Fraunhofer absorption lines as the reference. This method is different to previous study because only individual solar lines are used here instead of fitting the entire solar spectra. The ACGS has solar-viewing calibration through the diffuser over the North Pole. The offset can be straightforward calculated based on the observing actual locations of solar lines avoiding complex simulation and matching procedure of two spectra. But the disadvantage is that the result depends on the selected single positions of lines which can be affected by the uncertainty of radiometric calibration. The second method uses the entire atmospheric spectra as the reference to assess the accuracy of spectral calibration on orbit. This method is similar to previous study using fitting algorithm to fit the measured atmospheric spectra with the simulated spectra. The ACGS cloud-free simulation generated by radiative transfer model is used as the reference spectra. Both a shift and squeeze can be estimated by matching observation against the simulation spectra throughout the entire band. This method, not depending on single atmospheric lines, needs complex iterations or least-squares-fitting 
between observed and simulated spectra . And, calculation failure can take place sometimes if the simulation is insufficient accurate. Therefore, the two method complement each other.

\section{Spectral calibration using individual solar lines}

60 The TanSat ACGS instrument observes the Sun through a common diffuser to reduce the solar irradiance. The diffuser do not change the ILS because of even illumination of the telescope aperture. During the on-orbit testing from the February to July in 2017, measurements of the Sun are performed near the northern terminator once every two orbits, i.e. $6 \sim 7$ times per day. The routine solar observations are conducted only once a day after the on-orbit testing is finished. For a given orbit over the northern terminator, after the satellite finishes its science observations and before the satellite goes into the night, there are usually about 10 minutes for solar observations including 3 minutes for pointing preparation, 5 minutes for directly solar viewing by diffuser and the last 2 minutes for solar occultation observations. The middle 5 minutes solar measurements yield more than 1000 frames of solar spectra. After the solar spectra are corrected for the Doppler effect, they are merged into one single oversampled solar spectrum for evaluating spectral calibration accuracy.

\subsection{Method}

A two-dimensional FPA, one dimension for spatial field of view and one for spectrum, is used to collect the radiance for each band. The spectra are dispersed to illuminate all pixels in spectral dimension on each band. The numbers of pixels for $\mathrm{O}_{2}$ A-band, WCO2 and SCO2 are 1242, 500 and 500, respectively. In order to acquire s spectral sampling of more than two pixels per full width at half maximum (FWHM) in the range of spectrum and to get a better signal-to-noise ratio (SNR), the spectral resolution is decreased to $0.14 \mathrm{~nm}$ in $\mathrm{WCO} 2$ and $0.18 \mathrm{~nm}$ in SCO2 band, which are lower than that of OCO-2 (Frankenberg et al., 2014; Crisp et al., 2017). Table 1 shows the detailed spectral parameters of the TanSat ACGS instrument.

The TanSat ILS and wavelength of each pixel in three band were determined by a tunable diode laser before launch (Yang et al., 2018). Figure 1 shows the ILS profiles as examples at some middle pixels for three bands. The wavelength $\lambda_{p}$ for a single pixel is expressed by a fifth-degree polynomial as follows:

$\lambda_{p}=\sum_{i=0}^{5} c_{i} \times p^{i}$

80 where $p$ refers to the pixel number index ranging from the first pixel to the last pixel in each detector. The total number of pixels is shown in Table 1. The $c$ are the dispersion coefficients associating the spectral pixel index with its wavelength. These coefficients, which are different for each FOV within each band, are initially calculated by fitting the spectral pixel index and its associated wavelength at the laboratory before launch. An example of wavelength calculated by this formula at FOV 5 is shown in Figure 2. The accuracy of this parametrization is sufficient for ACGS's spectral calibration requirement that is one tenth of the spectral resolution.

The reference spectrum is taken from Kurucz solar spectrum atlas with different resolution available for scientific community (Fontenla et al., 1999; Chance and Kurucz, 2010). The Kurucz's spectra with the sampling resolution of $0.001 \mathrm{~nm}$ in the three 
bands are available via the internet (http://Kurucz.harvard.edu/sun). The Fraunhofer lines, which result from the absorption of sun light by elements in the outer layers of the Sun, can be clearly distinguished in the spectrum observed by ACGS pointing the Sun through the diffuser. The resolution of the Kurucz solar spectrum is one order of magnitude higher than that of ACGS. Therefore, the Kurucz solar spectrum is suitable for spectral calibration of ACGS on orbit.

In this method, individual solar lines, not the entire solar irradiance, are used as references. Figure 3 shows the Kurucz solar spectrum for each band. The positions of solar absorption lines are well determined in these spectra and can be used as the reference throughout the three ACGS's bands. The spectral calibration method using solar spectrum needs to select the suitable lines with its center position as the reference standard. These lines should be single and possess adequate intensity so that they can be easily distinguished from the spectrum. The wavelength offset with respect to the wavelength measured at ground is caused by the instrumental effects and Doppler effects on orbit. The latter can be calculated based on the relative velocity of the spacecraft and the Sun. The formula for Doppler correction $f_{d}$ is:

$f_{d}=f\left(1+V_{\text {rel }} / c\right)$

where $c$ is the speed of light, and $f$ is the raw solar irradiance frequency. The relative velocity $V_{\text {rel }}$ of the satellite and the Sun takes a positive value when they are approaching each other. The solar calibration are carried out at about $7 \mathrm{kms}^{-1}$ relative velocity that moves the spectra by $\sim 1 / 2$ spectral resolution of the $\mathrm{O}_{2} \mathrm{~A}$-band. After the Doppler correction is completed, these spectra are merged into one single oversampled solar spectrum. Then, the selected lines are compared to the expected positions to obtain the wavelength offset induced by the instrumental effects.

\subsection{Calibration result}

According to the criteria for the lines selection, we have selected ten, eight and eight solar lines in the $\mathrm{O}_{2} \mathrm{~A}-\mathrm{band}$, WCO2 and $\mathrm{SCO} 2$ band, respectively. Figure 3 shows high-resolution reference spectrum in three bands and the positions of the selected lines are also marked. These lines almost evenly distribute across the spectrum. In the phase of TanSat testing on orbit, we used these selected lines as reference for spectral calibration of the ACGS. Then, this method are successfully implemented in the operation monitoring and the raw data to level-1 product processing.

After Doppler correction in each pixel, we compare the typical Fraunhofer lines in the ACGS observation to the expected positions to obtain the offset in wavelength. Figure 4 - Figure 6 shows the temporal variation of wavelength offset of the measured solar spectra for all spatial FOVs in 2017. The mean offset of each FOV is about $-0.002 \mathrm{~nm}$ in $\mathrm{O}_{2}$ A-band, $-0.007 \mathrm{~nm}$ in WCO2 band and $-0.008 \mathrm{~nm}$ in SCO2 band, respectively. The wavelength offsets of each band have annually variation in a year. Their varying patterns are very similar for all FOVs. The ranges of variation are within $10 \%$ of spectral resolution which meets the accuracy requirement. There are little thermal gradient of the optics and FPAs. These thermal variation can result in the slight change of the geometry of major optical components such as collimator, grating and FPAs. Then, these geometric change can cause systematic offset which are similar for each FOV in each band. A noticeable increase on 24 May 2017 ( Day of Year (DOY) is 144) is caused by the switching of strategy of solar calibration, which is $6 \sim 7$ times per day during on-orbit testing period and once per day after that. This switching has significant influence on the whole thermal equilibrium. 
The statistics of offsets are calculated for each band, each spatial FOV, and each solar lines. Figure 7 - Figure 9 show the statistics of the offsets between measured solar lines and the reference lines in 2017. The largest standard deviations are observed in the $\mathrm{SCO} 2$ band, followed by the $\mathrm{WCO} 2$ band, with a much lower standard deviations in the $\mathrm{O}_{2} \mathrm{~A}$-band. The better statistical feature in $\mathrm{O}_{2}$ A-band is found, which we attribute to the insensitivity to thermal variations in this band. The offset for the SCO2 band is much noisier than the other two bands. Temperature variations have larger effects on the SCO2 bands than that on $\mathrm{O}_{2}$ A-band and $\mathrm{WCO} 2$ band. The $\mathrm{O}_{2}$ A-band uses the silicon detector to respond to radiance, while the two $\mathrm{CO}_{2}$ bands use the $\mathrm{HgCdTe}$ detector which are more sensitive to minor variations in temperature. These results demonstrate that these solar lines can be used as reference to determine the wavelength offset, although the solar lines are significantly weaker in the three band than those in UV-visible bands.

\section{Calibration with entire atmospheric spectra}

\subsection{Method}

A fitting method developed by Geffen and van Oss (2003) was applied to the recalibration of GOME spectra. And, Sun et al. (2017) also proposed the ILS fitting algorithm to characterize the OCO-2 ILS. Briefly, these methods use a high-resolution reference spectrum, i.e., solar spectrum therein, as the reference spectrum. Simulated spectrum is constructed by convolution of the high resolution reference spectrum and the ILS function. Two parameters, a shift $\alpha$ and a squeeze $\beta$ which are defined with respect to the raw dispersion coefficients, are determined by a best matching between the observed spectrum and the simulated spectrum. The shift applying to the first coefficient in (1) is equal for all pixels, whereas the squeeze applying to the second coefficient is related to the pixel position index. The difference between the measured spectrum and the simulated spectrum can be expressed by a function:

$140 f(\alpha, \beta)=\frac{1}{n-2} \sum_{i=1}^{n}\left[\frac{M(i)-S(i, \alpha, \beta)}{\delta M(i)}\right]^{2}$

where $\mathrm{n}$ is the number of pixels in each band; $M(i)$ and $S(i, \alpha, \beta)$ denotes the measured and simulated spectrum; and $\delta M(i)$ represent the uncertainty in the measurement. In this study, we use the search routine to find the minimum of equation (3).

In this section, the entire high-resolution atmospheric spectrum, not individual lines, is used as the reference spectrum for the ACGS on-orbit spectral calibration. The reference spectrum is simulated by the radiative transfer model which is the forward model of the TanSat retrieval algorithm for $\mathrm{XCO}_{2}$. Reanalysis data from ECMWF is used as the background in the simulation. The atmospheric absorption structures are involved in the reference spectrum. There are so large numbers of and complex absorption lines in the earthshine spectrum that the atmospheric absorption lines dominate over the solar absorption features which are obvious on the top of the atmosphere. Hence, the result of this method is independent of solar lines and can be compared with the calibration results shown in section 2.

150 The earthshine spectra observed by the ACGS are the products of the high-resolution spectrum and the ILS function for each pixels. In the procedure, the high resolution reference spectrum is degraded to the ACGS's resolution with the ILS. The 
common analytical functions (for example, Gauss-like function) could not fit the measured ILS very well. Figure 1 shows an example of ILS for each band, which is very similar to a normalized Gauss-like function. But at the top of ILS in the two $\mathrm{CO}_{2}$ bands, the ILS are a little broader than a Gaussian function, and at the bottom of ILS , there are significantly minor irregular structures. Therefore, the preflight ILS were given by lookup tables. Totally, ACGS has $1242 \times 9,500 \times 9$ and $500 \times 9$ different ILS tables in $\mathrm{O}_{2}$ A-band, WCO2 and SCO2 band, respectively. The ACGS ILS functions have full widths at half maximum (FWHM) resolutions of $0.04 \mathrm{~nm}$ for $\mathrm{O}_{2}$ A-band, $0.14 \mathrm{~nm}$ for WCO2 band and $0.18 \mathrm{~nm}$ for SCO2 band (see Table 1). Mathematically, the convolved reference spectrum at a detector pixel with a central wavelength $\lambda$ is described by

$I(\lambda)=\int I\left(\lambda^{\prime}\right) \times I L S\left(\lambda, \lambda^{\prime}\right) d \lambda^{\prime}$

where the $\lambda^{\prime}$ integration is on the full range of the ILS function. The $I(\lambda)$ is referred to as the reference spectrum for spectral calibration of ACGS on orbit.

\subsection{Calibration result}

This scheme is applied as a complement and verification for the solar calibration because this scheme strongly depends on reanalysis data, which acquisition has a very long delay, at least 1 month from ECMWF. The wavelength shift and squeeze with respect to initial central wavelength are estimated by applying this scheme over the three bands assuming the ILS functions are constant. The initial wavelength from which the calibration starts is the same as that used in the solar calibration, so that the result can be compared with that from solar calibration.

We perform this calibration for many different orbits, and find that the pattern of the variation along one orbit is very similar. As an example, Figure 10 shows wavelength shift terms along latitude for the nadir observation in the entire orbit on 23 April 2017. These spectra on this orbit are suitable for evaluating the spectral calibration in detail because most of observations are acquired in cloud-free scenes. The changing patterns for each FOV are very similar in the $\mathrm{O}_{2}$ A-band and WCO2 band. But the inter-FOV differences in the $\mathrm{SCO} 2$ band are significant and there is no clear pattern of changing for each FOV in this band. Table 2 shows the statistics of the shifts for the nine FOVs in each band. The shift variations in the $\mathrm{O}_{2}$ A-band are more stable than that in the other two bands. Also, the variations are very close and similar for nine FOVs. These statistical features show larger differences for the nine FOVs in SCO2 band.

The shifts derived from this method agree closely with that calculated from solar spectra. The mean shift is larger than the offset calculated from the solar calibration. The larger shift derived by applying earthshine spectra mainly come from the uncertainties in the simulation using radiative transfer model. For instance, the surface albedo and the optical properties of the atmosphere or aerosols can influence the depths of the absorption lines, and then influence the fitting between the measurement and simulation. Other possible reasons lies in the fact that most of the SNR in the earthshine spectra is lower than that in the solar spectra, particularly for the SCO2 band.

Figure 11 shows the example of matching the measurement and simulation spectra by applying shift and applying shift together with squeeze in the $\mathrm{O}_{2}$ A-band. The measured spectrum is shifted by about $0.005 \mathrm{~nm}$ to get a better agreement with the simulated spectrum. This quantity is about $12 \%$ of the spectral resolution, while the squeeze term is approximately $0.07 \%$ 
of the spectral resolution. The squeeze term can be neglected because the effect on the wavelength variation is very small compared against that of the shift. The mechanical tilt of FPAs can leads to obvious squeeze term in wavelength (Crisp et al., 2017). Hence, the tilts for FPAs are almost negligible relative to the optical axis after launch.

\section{Conclusions}

This study applies two methods of wavelength calibration to evaluate the wavelength change of the TanSat ACGS instrument. In addition to monitor spectral stability, both schemes can be used to gain spectral knowledge prior to the product processing. The ACGS observes the Sun through a common diffuser which reflects the sunlight into the telescope aperture. In each band for the solar spectra, the individual and complete solar lines including the wings on both sides of the lines are selected to calculate the wavelength offset. A high resolution solar spectrum is used to provide the actual position as the reference standard for each selected line. The offsets calculated by each line show better agreements with each other. The accuracy of wavelength offsets is better than $10 \%$ of the FWHM that meet the requirements of spectral calibration of the ACGS on orbit. In addition, the simulated entire earthshine spectrum is used as the reference to derive the shift and squeeze terms by fitting the measured and the modelled spectra. The shifts derived from this method agree closely with that calculated from solar spectra. The changes of wavelengths both show the best stabilities and the smallest inter-FOV variations in $\mathrm{O}_{2}$ A-band, followed by the WCO2, and then by SCO2 band for the two method. This appearance may be induced by the different sensitivities to temperature variation on FPA in each band. Therefore, it is necessary to simultaneously estimate the minor shift along with other parameters in the full physics retrieval algorithm for $\mathrm{X}_{\mathrm{CO}_{2}}$.

Data availability. The results presented here are based on TanSat L1B solar calibration data, which are publicly available via the internet at the site http://satellite.nsmc.org.cn/portalsite/default.aspx.

Author contributions. Yanmeng Bi, Qian Wang and Zhongdong Yang performed all calculations for this paper together with all coauthors. Chengbao Liu helped with the geophysical and Doppler shift calculation. Chao Lin and Longfei Tian helped with the analysis of the change of wavelength on orbit. Naiqiang Zhang, Yanpin Luo and Yacheng Wang helped with the raw data processing.

Competing interests. The authors declare that they have no conflict of interest.

Acknowledgements. We would like to thank Kurucz for the high resolution solar spectra. We also thank ECMWF for the reanalysis data for our simulation. The research described in this paper was carried out at NSMC under a contract (2011AA12A104) as part of a major project of the Ministry of Science and Technology (MOST), China Earth Observation Program. 


\section{References}

Chance, K.: Analysis of BrO measurements from the Global Ozone Monitoring Experiment, Geophysical Research Letters, 25, 3335-3338, https://doi.org/10.1029/98GL52359, 1998.

Chance, K. and Kurucz, R. L.: An improved high-resolution solar reference spectrum for earth's atmosphere measurements in the ultraviolet, visible, and near infrared, Journal of Quantitative Spectroscopy \& Radiative Transfer, 111, 1289-1295, 2010.

Chatterjee, A., Gierach, M. M., Sutton, A., Feely, R. A., Crisp, D., Eldering, A., Gunson, M. R., Odell, C. W., Stephens, B. B., and Schimel, D. S.: Influence of El Niño on atmospheric $\mathrm{CO}_{2}$ over the tropical Pacific Ocean: Findings from NASA's OCO-2 mission, Science, 358 , 2017.

Crisp, D., Fisher, B., Odell, C. W., Frankenberg, C., Basilio, R., Bosch, H., Brown, L. R., Castano, R., Connor, B. J., Deutscher, N. M., et al.:

The ACOS $\mathrm{CO}_{2}$ retrieval algorithm - Part II: Global $\mathrm{X}_{\mathrm{CO}_{2}}$ data characterization, Atmospheric Measurement Techniques, 5, 687-707, 2012.

Crisp, D., Pollock, H. R., Rosenberg, R., Chapsky, L., Lee, R. A. M., Oyafuso, F. A., Frankenberg, C., O’Dell, C. W., Bruegge, C. J., Doran, G. B., Eldering, A., Fisher, B. M., Fu, D., Gunson, M. R., Mand rake, L., Osterman, G. B., Schwandner, F. M., Sun, K., Taylor, T. E., Wennberg, P. O., and Wunch, D.: The on-orbit performance of the Orbiting Carbon Observatory-2 (OCO-2) instrument and its radiometrically calibrated products, Atmospheric Measurement Techniques, 10, 59-81, https://doi.org/10.5194/amt-10-59-2017, 2017.

Fontenla, J., White, O. R., Fox, P., Avrett, E. H., and Kurucz, R. L.: Calculation of Solar Irradiances. I. Synthesis of the Solar Spectrum, The Astrophysical Journal, 518, 480-499, 1999.

Frankenberg, C., Pollock, R., Lee, R. A. M., Rosenberg, R., Blavier, J. F., Crisp, D., Apos, C. W. O., Dell, Osterman, G. B., Roehl, C. M., et al.: The Orbiting Carbon Observatory (OCO-2): spectrometer performance evaluation using pre-launch direct sun measurements, Atmospheric Measurement Techniques, 8, 301-313, 2014.

Geffen, J. H. G. M. V. and van Oss, R. F.: Wavelength calibration of spectra measured by the Global Ozone Monitoring Experiment by use of a high-resolution reference spectrum, Appl. Opt., 42, 2739-2753, 2003.

Liu, X., Chance, K., Sioris, C. E., Spurr, R. J. D., Kurosu, T. P., Martin, R. V., and Newchurch, M. J.: Ozone profile and tropospheric ozone retrievals from the Global Ozone Monitoring Experiment: Algorithm description and validation, Journal of Geophysical Research (Atmospheres), 110, D20307, https://doi.org/10.1029/2005JD006240, 2005.

Liu, X., Bhartia, P. K., Chance, K., Spurr, R., and Kurosu, T. P.: Ozone profile retrievals from the Ozone Monitoring Instrument, Atmospheric Chemistry and Physics, 10, 2521-2537, 2010.

Miller, C., Crisp, D., DeCola, P., Olsen, S., Randerson, J., Michalak, A., Alkhaled, A., Rayner, P., Jacob, D., Suntharalingam, P., Jones, D., Denning, S., Nicholls, M., Doney, S., Pawson, S., Boesch, H., Connor, B., Fung, I., O’Brien, D., and Law, R.: Precision requirements for space-based XCO2 data, Journal of Geophysical Research, 112, 2007.

Munro, R., Lang, R., Klaes, D., Poli, G., Retscher, C., Lindstrot, R., Huckle, R., Lacan, A., Grzegorski, M., Holdak, A., Kokhanovsky, A., Livschitz, J., and Eisinger, M.: The GOME-2 instrument on the Metop series of satellites: instrument design, calibration, and level 1 data processing - an overview, Atmospheric Measurement Techniques, 9, 1279-1301, 2016.

O’Dell, C., Connor, B., Boesch, H., O’Brien, D., Frankenberg, C., Castaño, R., Christi, M., Crisp, D., Eldering, A., Fisher, B., Gunson, retrieval algorithm - Part 1: Description and validation against synthetic observations, Atmospheric Measurement Techniques, 5, -121 , https://doi.org/10.5194/amtd-4-6097-2011, 2012. 
https://doi.org/10.5194/amt-2020-20

Preprint. Discussion started: 20 May 2020

(c) Author(s) 2020. CC BY 4.0 License.

(c) (1)

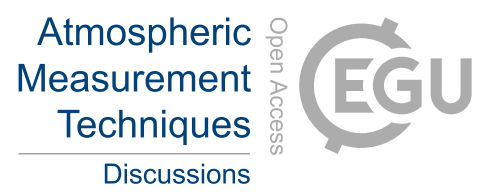

Schwandner, F. M., Gunson, M. R., Miller, C. E., Carn, S. A., Eldering, A., Krings, T., Verhulst, K. R., Schimel, D. S., Nguyen, H. M., Crisp, D., et al.: Spaceborne detection of localized carbon dioxide sources., Science, 358, 2017.

250 Sun, K., Liu, X., Nowlan, C. R., Cai, Z., Chance, K., Frankenberg, C., Lee, R. A. M., Pollock, R., Rosenberg, R., and Crisp, D.: Characterization of the OCO-2 instrument line shape functions using on-orbit solar measurements, Atmospheric Measurement Techniques, 10, 939-953, https://doi.org/10.5194/amt-10-939-2017, 2017.

Yang, Z., Zhen, Y., Yin, Z., Lin, C., Bi, Y., Liu, W., Wang, Q., Wang, L., Gu, S., and Tian, L.: Laboratory spectral calibration of the TanSat atmospheric carbon dioxide grating spectrometer, Geoscientific Instrumentation, Methods and Data Systems, 7, 245-252, https://doi.org/10.5194/gi-7-245-2018, 2018.

Yang, Z., Bi, Y., and Q., W.: Inflight Performance of the TanSat Atmospheric Carbon Dioxide Grating Spectrometer, IEEE Trans. Remote Sens., Accepted, 2019. 


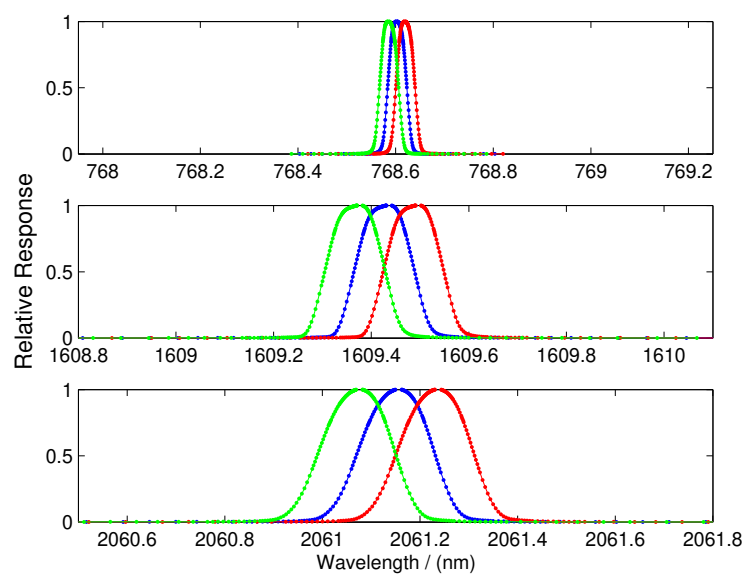

Figure 1. The preflight ILS function at three adjacent pixel located in the central section of the FPA for three band. The top panel is for the $\mathrm{O} 2 \mathrm{~A}$, the middle panel is for $\mathrm{WCO} 2$ and the bottom panel is for $\mathrm{SCO} 2$.

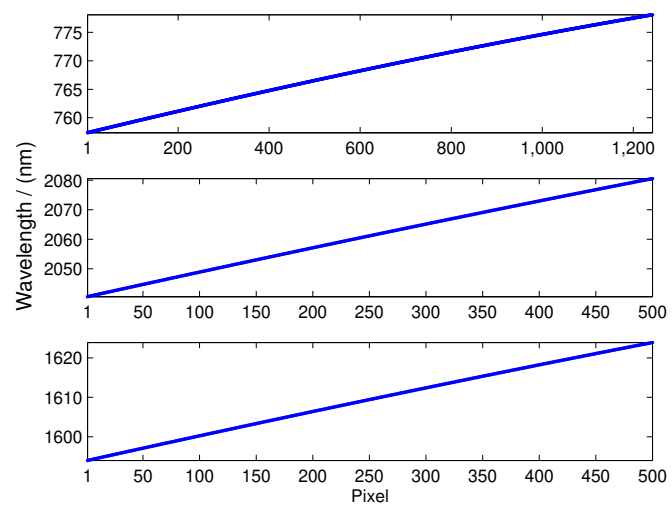

Figure 2. An example of wavelength as function of pixel index in the focal plane at FOV 5 for three band. The panels for three bands are similar to Fig. 1 


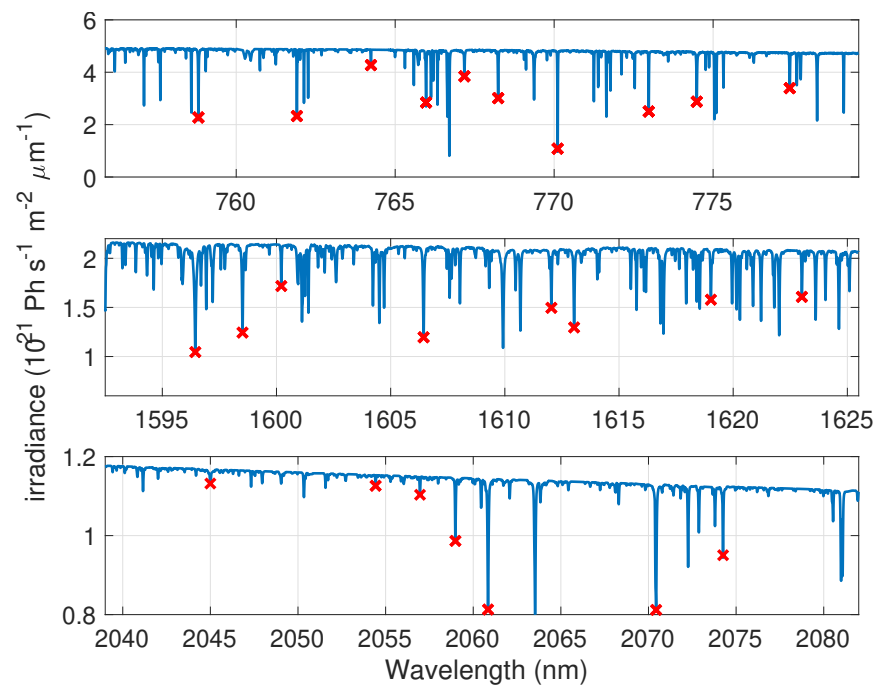

Figure 3. The Kurucz solar spectra and the positions of solar absorption lines selected as reference in three band, labelled separately by red cross. The panels for three bands are similar to Fig. 1

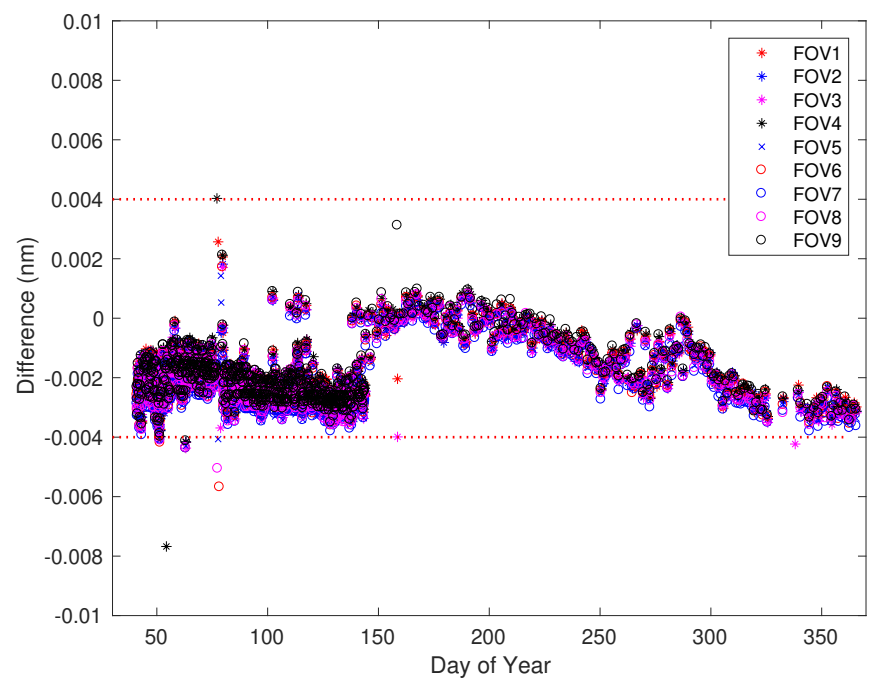

Figure 4. The time series of wavelength change of the measured solar lines for 9 spatial FOVs in $\mathrm{O}_{2}$ A-band. The dashed red lines show the $10 \%$ of the spectral resolution in $\mathrm{O}_{2}$ A-band, i.e., $\pm 0.004 \mathrm{~nm}$. 
https://doi.org/10.5194/amt-2020-20

Preprint. Discussion started: 20 May 2020

(c) Author(s) 2020. CC BY 4.0 License.

(c) (i)
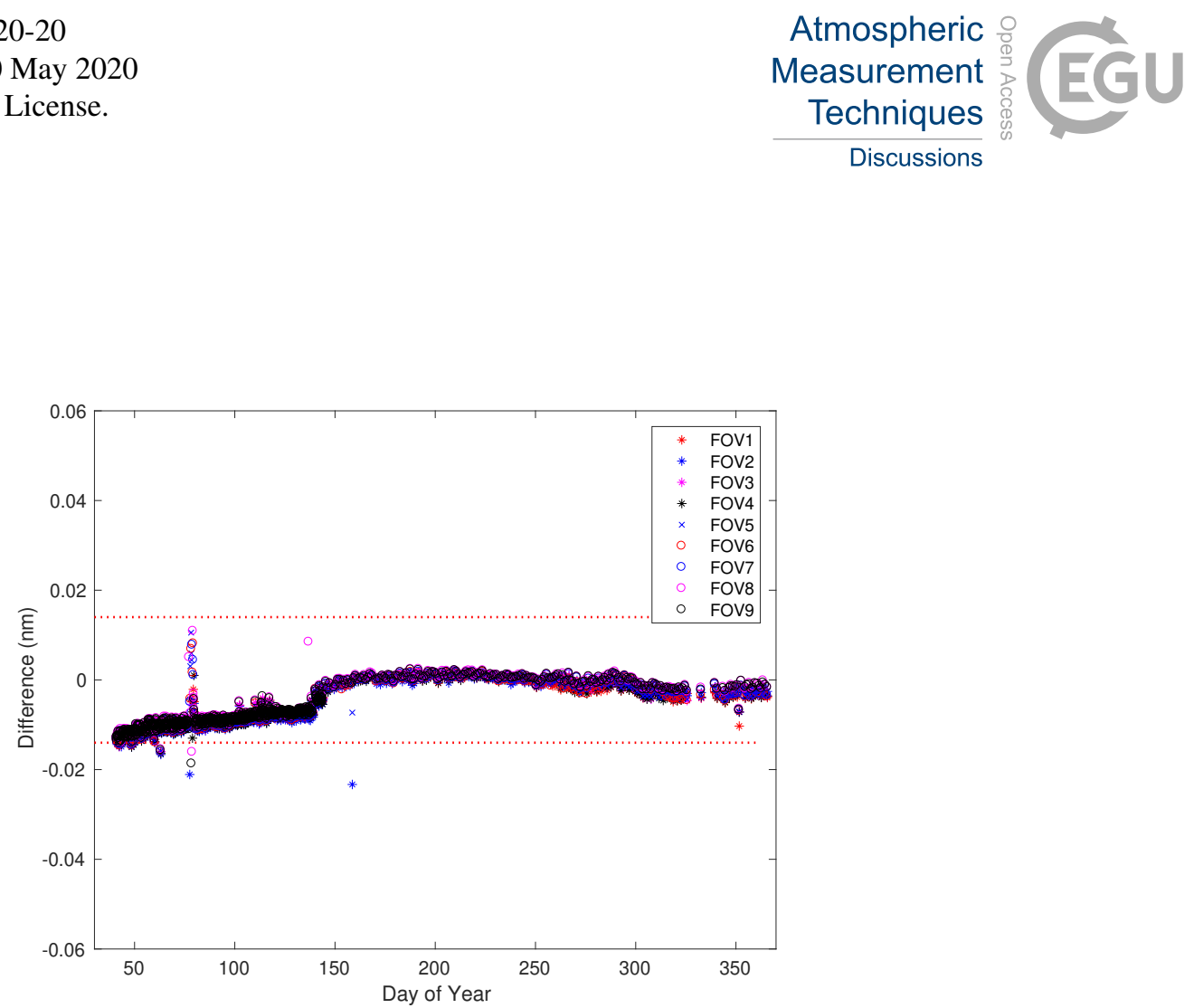

Figure 5. The time series of wavelength change of the measured solar lines for 9 spatial FOVs in $\mathrm{WCO} 2$ band. The dashed red lines show the $10 \%$ of the spectral resolution in WCO2 band, i.e., $\pm 0.014 \mathrm{~nm}$.

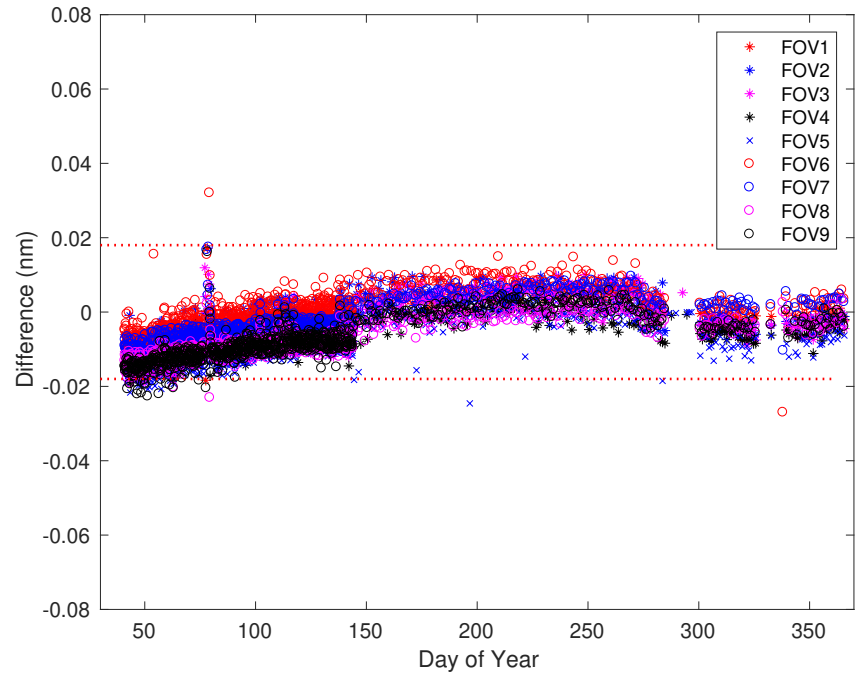

Figure 6. The time series of wavelength change of the measured solar lines for 9 spatial FOVs in SCO2 band. The dashed red lines show the $10 \%$ of the spectral resolution in WCO2 band, i.e., $\pm 0.018 \mathrm{~nm}$. 


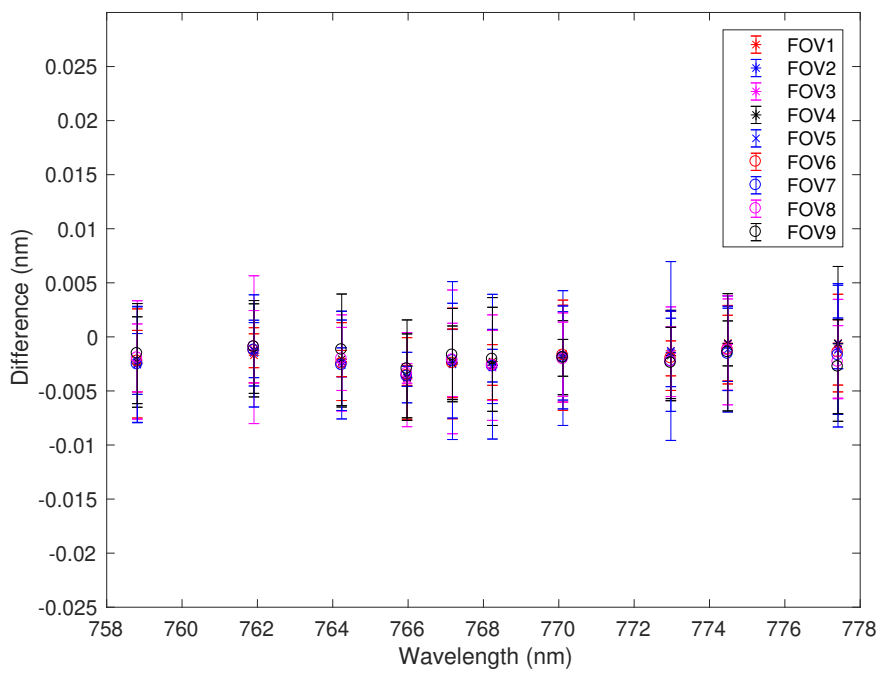

Figure 7. The statistics of wavelength change on the locations of 9 solar lines for all spatial FOVs in $\mathrm{O}_{2}$ A-band.

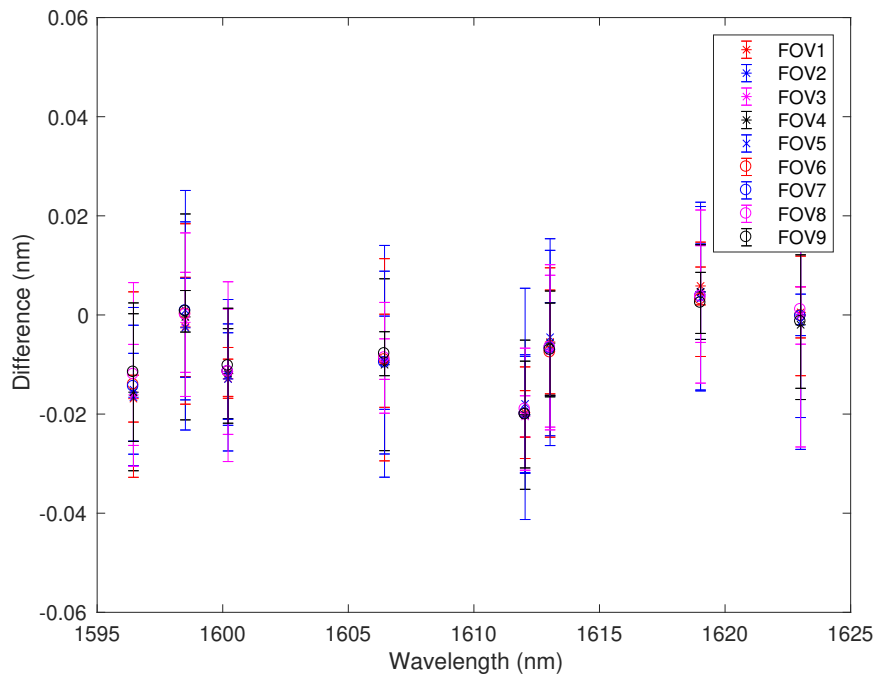

Figure 8. The statistics of wavelength change on the locations of 9 solar lines for all spatial FOVs in WCO2 band. 
https://doi.org/10.5194/amt-2020-20

Preprint. Discussion started: 20 May 2020

(c) Author(s) 2020. CC BY 4.0 License.

Atmospheric Measurement

Techniques

(c) (i)

Discussions

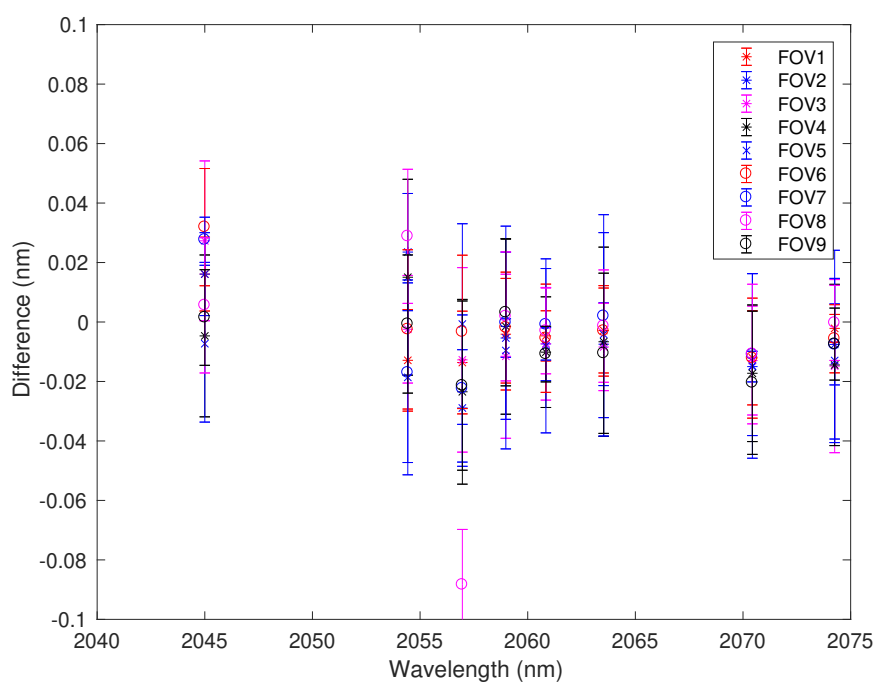

Figure 9. The statistics of wavelength change on the locations of 9 solar lines for all spatial FOVs in SCO2 band.

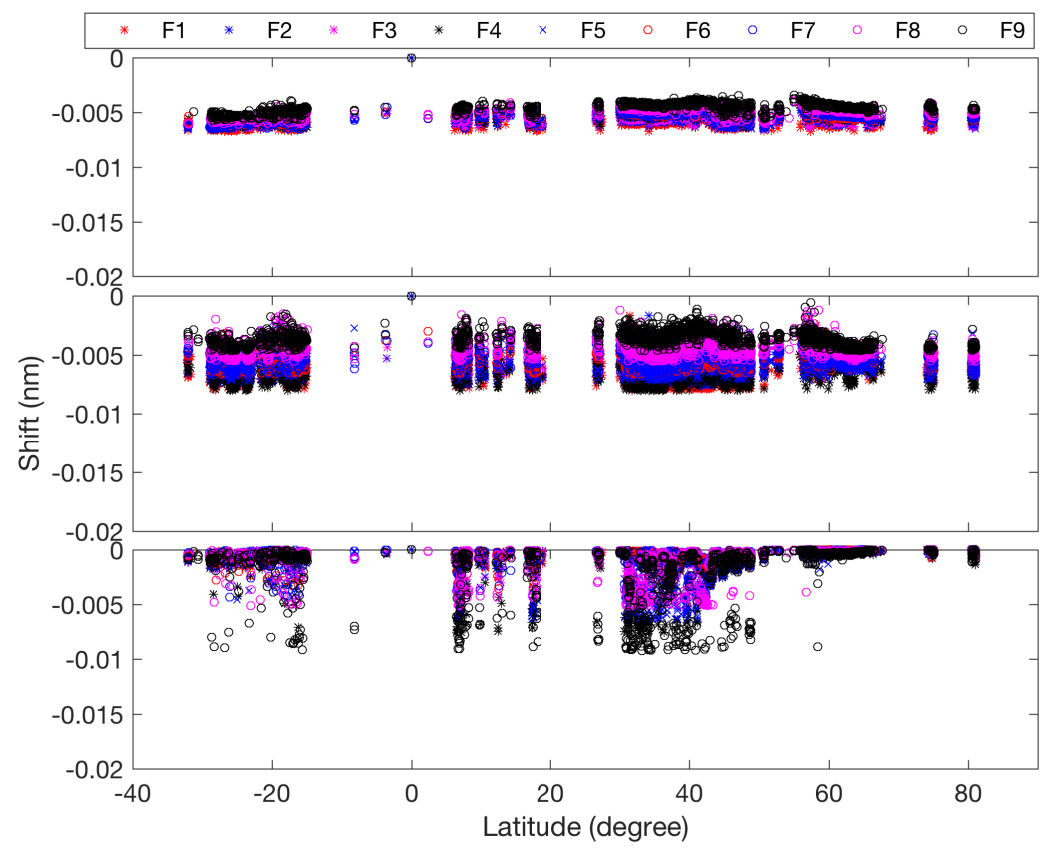

Figure 10. The wavelength change of each FOV along latitude for three band in one orbit on April 23, 2017 (DOY 113 ). 
https://doi.org/10.5194/amt-2020-20

Preprint. Discussion started: 20 May 2020

(c) Author(s) 2020. CC BY 4.0 License.

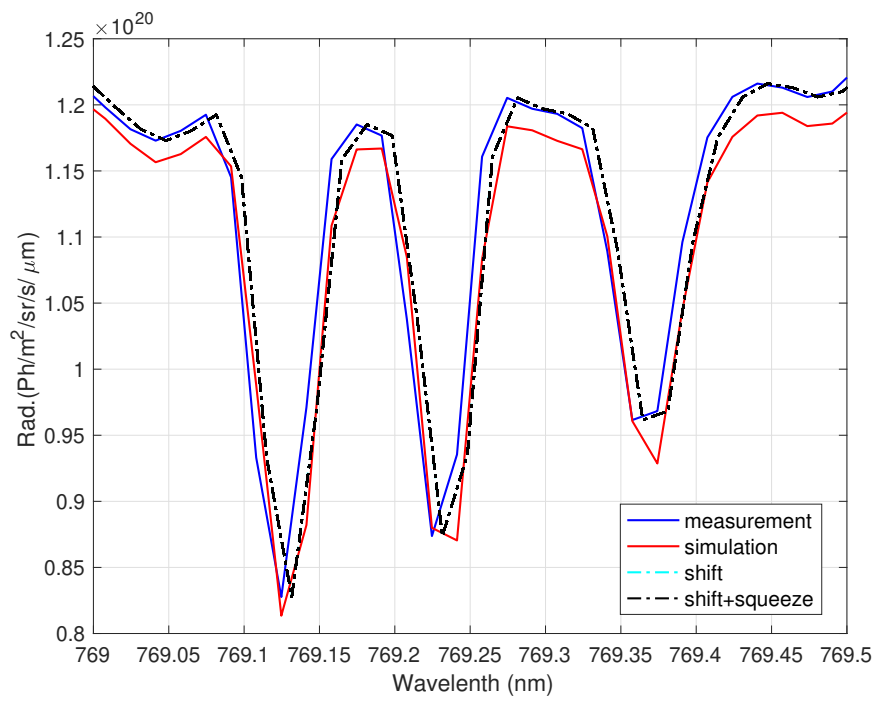

Figure 11. Example of wavelength calibration of TanSat ACGS for FOV 2. The cyan dashed line denotes the measurement spectra shifted. The black dashed line reprensents the measurement spectra shifted plus squeezed. The latter overlays the previous due to the very small squeeze. 
Table 1. Spectral parameters of the TanSat ACGS instrument

\begin{tabular}{cccc}
\hline Parameters & O2A & WCO2 & SCO2 \\
\hline Spectral range (nm) & $758-778$ & $1594-1624$ & $2042-2082$ \\
Resolution (nm) & $0.033-0.047$ & $0.12-0.14$ & $0.16-0.18$ \\
Spectral pixels & 1242 & 500 & 500 \\
Sampling / FWHM & $>2$ & $>2$ & $>2$ \\
FOV num. & 9 & 9 & 9 \\
\hline
\end{tabular}

Table 2. The mean and the standard deviation (Std.) of the change in the wavelength for each band using the atmospheric spectra calibration on 23 April 2017. (unit: $10^{-3} \mathrm{~nm}$ )

\begin{tabular}{|c|c|c|c|c|c|c|}
\hline \multirow{2}{*}{ FOV } & \multicolumn{2}{|c|}{$\mathrm{O} 2 \mathrm{~A}$} & \multicolumn{2}{|c|}{ WCO2 } & \multicolumn{2}{|c|}{$\mathrm{SCO} 2$} \\
\hline & Mean & Std. & Mean & Std. & Mean & Std. \\
\hline 1 & -5.928 & 0.205 & -7.022 & 0.559 & -0.523 & 0.424 \\
\hline 2 & -5.705 & 0.204 & -6.867 & 0.516 & -0.613 & 0.453 \\
\hline 3 & -5.580 & 0.226 & -6.099 & 0.505 & -0.742 & 0.636 \\
\hline 4 & -5.374 & 0.211 & -6.583 & 0.577 & -1.270 & 1.950 \\
\hline 5 & -5.352 & 0.184 & -5.808 & 0.537 & -1.281 & 1.854 \\
\hline 6 & -5.181 & 0.222 & -5.443 & 0.466 & -0.563 & 0.806 \\
\hline 7 & -5.188 & 0.215 & -5.319 & 0.444 & -0.870 & 1.355 \\
\hline 8 & -4.809 & 0.212 & -4.442 & 0.425 & -1.136 & 1.675 \\
\hline 9 & -4.449 & 0.219 & -3.653 & 0.376 & -2.116 & 3.038 \\
\hline
\end{tabular}

